Dorothea Schuller

\title{
Männlich, weiblich, was ihr wollt: Shakespeare und die Lust am Spiel mit den Geschlechtern
}

You've got your mother in a whirl She's not sure if you're a boy or a girl

David Bowie, „Rebel, Rebel“

„Männlich oder weiblich ist die erste Unterscheidung, die Sie machen, wenn Sie mit einem anderen menschlichen Wesen zusammentreffen, und Sie sind gewöhnt, diese Unterscheidung mit unbedenklicher Sicherheit zu machen." Mit diesen Worten richtete sich Sigmund Freud 1933 in seiner Vorlesung „Die Weiblichkeit“ an seine Zuhörer, nur um sie gleich anschließend darauf hinzuweisen, dass trotz dieser scheinbar ganz offensichtlichen Aufteilung der Menschen in Männer und Frauen, die wir tagtäglich geradezu reflexartig wiederholen, jedes Individuum Teile beider Geschlechter sowohl an seinem Körper als auch in seiner psychischen Konstitution trage (120-21). Außerdem warnte Freud vor dem Gebrauch der Adjektive ,männlich“ und ,weiblich', um damit Verhaltensformen zu bezeichnen, welche man ebenso gut mit den Begriffen ,aktiv` und ,passiv' beschreiben könne. 
$\mathrm{Zu}$ unterschiedlich seien die Charaktereigenschaften individueller Menschen, als dass man von den primären und vorwiegenden sekundären Geschlechtsmerkmalen eindeutige Schlussfolgerungen auf das Verhalten ziehen könne. Auch sei der, wie Freud es nennt, „Einfluss der sozialen Ordnungen“ (123), also der Einfluss von Kultur, auf die Ausbildung der Psyche und somit auch auf die Geschlechtsidentität noch so unerforscht, dass voreilig aufgestellte Essentialismen unbedingt zu vermeiden seien. Das ist nicht mehr der Freud des oft zitierten Ausspruchs „die Anatomie ist das Schicksal“ („Untergang“, 400).. Seine Ausführungen zur weiblichen Sexualentwicklung leitet Freud dann mit folgendem Satz ein: „Die Eigenart der Psychoanalyse entspricht es [...], dass sie nicht beschreiben will, was das Weib ist, - das wäre für sie eine kaum lösbare Aufgabe, - sondern untersucht, wie es wird“ („Weiblichkeit“, 124). Dieser Satz erinnert (erstaunlicherweise?) an Simone de Beauvoirs zentrale These ihres feministischen Manifests Le deuxième sexe (Das andere Geschlecht) von 1949: „Man kommt nicht als Frau zur Welt, man wird es“ (334). Mich beschäftigt hier nicht das „Rätsel der Weiblichkeit“ (,Weiblichkeit“, 120), das Freud vergeblich zu lösen suchte, sondern das Rätsel der Geschlechterdifferenz schlechthin und seine spielerische Thematisierung in Shakespeares Komödien.

Dass Definitionen von Geschlecht und mit Geschlecht assoziierten Verhaltensweisen stark kulturabhängig sind, ist die grundlegende Erkenntnis der Geschlechterforschung. Sie interessiert sich dafür, wie Weiblichkeit und Männlichkeit durch politische, juristische, medizinische, religiöse sowie literarische Diskurse verstanden und konstruiert werden. Als ein besonders fruchtbares Feld für Fragestellungen dieser Art hat sich für Literatur- und Kulturwissenschaftler dabei das dramatische und poetische Werk William Shakespeares herausgestellt, der vor allem in seinen Komödien erstaunlich aktuell wirkende Spekulationen über geschlechtliche Ambivalenz und die (theatralische) Repräsentation von gender anstellt.

Nach einer kurzen Einführung in die Fragen der Geschlechterforschung und einem Blick auf die elisabethanische Theaterpraxis werde ich mich den beiden Dramen Wie es euch gefällt und Was ibr wollt zuwenden, in denen Shakespeare das Spiel mit den Geschlechtern am offensichtlichsten und lustvollsten betreibt. Anschließend werde ich zwei Beispiele aus der produktiven Rezeptionsgeschichte dieser beiden Komödien vorstellen, die deren zentrale Themen in neue Kontexte stellen und kreativ kommentieren. Das ist zum einen Théophile Gautiers Roman Mademoiselle de Manpin aus dem frühen 19. Jahrhundert und zum anderen John Maddens erfolgreicher Film Shakespeare in Love aus dem Jahre 1998.

Mit dem aus der Grammatik übernommenen Begriff gender (die deutsche Entsprechung, Genus' hat sich nicht wirklich durchsetzen können) wird das soziale Geschlecht eines Menschen bezeichnet. Dieses ist in seiner Bedeutung wandelbar

1 Diese Abwandlung eines Napoleon-Zitates („Politik ist Schicksal“) benutzte Freud mehrfach, z.B. 1924 in „Der Untergang des Ödipuskomplexes“. 
und muss nicht notwendigerweise mit dem biologischen Geschlecht übereinstimmen. Denn was genau heißt es eigentlich, männlich oder weiblich zu sein? Welche Eigenschaften muss man aufweisen, um als männlich oder weiblich anerkannt zu werden? Und sollte Anatomie doch ausschlaggebend sein, warum wird dann von ,echten` Männern oder, unweiblichen' Frauen gesprochen, wenn es um Fragen des Verhaltens geht? Wer bestimmt, wo die Grenzen gezogen werden? Wo hört Natur auf und fängt Kultur an? Ist es vielleicht hilfreich, Gender als eine Art von performance anzusehen, eine Rolle, die wir bewusst oder unbewusst spielen, um bestimmte Signale an unser Gegenüber zu senden? Diese These wurde Anfang der 1990er Jahre besonders von der amerikanischen Philosophin Judith Butler angeregt, ${ }^{2}$ die außerdem darauf hinwies, dass es eigentlich unsinnig sei, nur zwei Gender-Auswahlmöglichkeiten anzubieten, wenn doch das soziale Geschlecht gerade nicht an das biologische gekoppelt ist, für das zudem ebenfalls mehr als zwei Kategorien bereitgestellt werden sollten. Und wie verhält es sich mit der Liebe? Ist das eigene Geschlecht ausschlaggebend für die Partnerwahl? Oder die eigene Geschlechterrolle? Was lässt eine andere Person attraktiv wirken? All diese Fragen, die uns noch immer umtreiben, werden bei Shakespeare auf unterhaltsame Art und Weise angerissen, ohne dass man ihn dafür anachronistisch lesen oder zu einem hellsichtigen Propheten machen müsste.

Trotz (oder vielleicht gerade wegen) der scheinbar so offensichtlichen Zweigeschlechtlichkeit, die wir im Alltag wahrnehmen, fühlten sich Mythos, Religion, Kunst und Literatur - wenn auch aus sehr unterschiedlichen Gründen - schon immer von Fällen angezogen, bei denen die Unterscheidung zwischen männlich und weiblich nicht so einfach zu treffen ist oder in denen eine Verschmelzung der vermeintlichen Gegensätze stattgefunden hat. Den androgynen Urmenschen findet man in vielen Schöpfungsgeschichten. Aus Ovids Metamorphosen bekannt ist der Mythos von Hermaphroditos, Sohn von Hermes und Venus, der gegen seinen Willen von der Nymphe Salmacis umworben wurde und nach deren Bitte an die Götter, sie möge nie von ihm getrennt werden, mit ihrem Körper verschmolz. In der Alchemie symbolisiert der doppelgeschlechtliche Hermaphrodit als Zeichen der Vollkommenheit den Stein der Weisen, und Geschichten über sich zum Verwechseln ähnlich sehende Bruder-Schwester-Paare sind fester Bestandteil mythologischer und literarischer Tradition. ${ }^{3}$ Aus der Popgeschichte ist das schillernde Zwitterwesen sowieso nicht mehr wegzudenken, man denke nur an David Bowie, K. D. Lang oder Bill Kaulitz, den Sänger der Gruppe Tokio Hotel.

2 Die deutsche Übersetzung von Butlers einflussreichem Buch Gender Trouble erschien 1991 unter dem Titel Das Unbehagen der Geschlechter.

3 Es ist wichtig, hier zwischen Mythos und historischer Realität zu trennen: Tatsächliche Hermaphroditen (heute bevorzugt man den Begriff ,Intersexuelle) wurden in den meisten Gesellschaften marginalisiert oder zur Assimilation, also zur Entscheidung für ein Geschlecht, gezwungen. Vgl. Barbin / Foucault. 
Dass das Spiel mit den Geschlechtern sowohl intellektuell wie erotisch anregend ist, hat auch Shakespeare erkannt, und die gleiche Atmosphäre von Magie und Sinnlichkeit, die oft mit dieser Thematik verbunden ist, findet sich auch in mehreren seiner Komödien. Trotz häufiger Verweise auf mythologische Quellen greift Shakespeare jedoch stets zum gleichen Mittel, um seine Figuren zumindest für eine Zeit lang aus ihrer eindeutigen Geschlechtszugehörigkeit herauszulösen: Nicht durch göttliches Eingreifen oder Zauberei, sondern durch einen schlichten Kleidungswechsel verwandeln sich gleich eine ganze Reihe seiner Heldinnen in gutaussehende junge Männer. Zudem haben sie alle das notwendige schauspielerische Talent, um die ungewohnte Rolle glaubwürdig zu verkörpern. Tatsächlich fällt der Rollentausch so leicht, und vor allem Rosalind ${ }^{4}$ in Wie es euch gefällt hat solchen Spaß in ihrem männlichen Part, dass der Wechsel von Frau zu Mann einerseits ganz natürlich wirkt und andererseits gerade dadurch die grundlegende Künstlichkeit einer essentialistisch gedachten Geschlechtsidentität herausstreicht. Warum sollte es etwa einem Mädchen nicht möglich sein, sich frech und schlagfertig zu geben oder mit einem anderen Mädchen zu flirten? Für die Dauer des Stücks haben diese Shakespeare-Heldinnen die Möglichkeit, die im 16. Jahrhundert streng patrouillierte Grenze zwischen männlich und weiblich zu durchbrechen. Somit geschieht das Verkleidungsspiel nicht etwa zum alleinigen Zwecke der Komik, die sich aus den resultierenden Missverständnissen ergibt, sondern kann durchaus als lustvolle Aufhebung aller eindeutigen Zuschreibungen gesehen werden. Die transvestierten Heldinnen sind nicht einfach nur Frauen in Männerkleidern, sondern dienen dazu, eine Art drittes Element in die bestehende Zweigeschlechtlichkeit einzuführen, das sowohl auf Männer als auch auf Frauen anziehend wirkt. So bringt die als Page verkleidete Viola in Was ibr wollt die Handlung überhaupt erst ins Rollen, als sie vom Herzog Orsino den Auftrag bekommt, in seinem Namen um die schöne Olivia zu werben. Diese verliebt sich Hals-überKopf in den vermeintlichen Jüngling, während sich Viola selbst zu Orsino hingezogen fühlt, der von seinem neuen Höfling ebenfalls sehr angetan ist. Dass das elisabethanische Publikum diese Form des gender bending - also das Verunklaren der Geschlechterdifferenz - zu schätzen wusste, mag man aus den Titeln der beiden berühmtesten Komödien dieser Art ablesen: Wie es euch gefällt und Was ibr wollt (im Englischen As You Like It und Twelfth Night, or What You Will). In beiden Stücken stehen Figuren im Mittelpunkt, die durch ihre mann-weibliche Doppelrolle für Vergnügen und erotische Verwirrung sorgen. Weitere Frauen in Männerkleidern sind zudem in Zwei Herren aus Verona, Der Kaufmann von Venedig und Cymbeline zu finden.

Wie sehr das Verhältnis von biologischem Geschlecht und Gender, also den sozio-kulturellen Erscheinungsformen von Männlichkeit und Weiblichkeit, historischem Wandel unterworfen ist, lässt sich gerade am Phänomen des cross-dressing

$4 \quad$ Im Folgenden wird die deutsche Version diese Namens - Rosalinde - verwendet. 
sehr gut aufzeigen und soll anhand einer Alltagsbeobachtung kurz verdeutlicht werden. Die meisten Menschen wissen aus Gewohnheit beim Anblick der simplen Piktogramme an öffentlichen Toiletten, die die Geschlechterdifferenz auf Rock versus Hose reduzieren, wo sie sich zuzuordnen haben. Doch stellen wir uns einen Außerirdischen vor, der eine durchschnittliche Gruppe von Menschen des 21. Jahrhunderts den beiden Symbolen zuordnen sollte - die Damentoilette wäre seltsam verwaist. Wir sehen, Kleidung als äußerliches Merkmal von Geschlechtszugehörigkeit zu betrachten, ist eine sehr ungenaue Methode, um eindeutige Unterscheidungen vorzunehmen. Mode ist kulturabhängig und hat mit dem biologischen Geschlecht nur selten etwas zu tun. ${ }^{5}$ Mit der Einführung der Hose als akzeptables Kleidungsstück für Frauen wurde die Kostümfrage bei modernen Inszenierungen von Shakespeares cross-dressing comedies plötzlich zum Problem. Das Kleid gegen Jackett und Hose auszutauschen, ist mittlerweile auf der Zeichenebene nicht mehr ausreichend, um die ,Verwandlung' einer Frau in einen Mann zu signalisieren. ${ }^{6}$ Dies mag der Grund dafür sein, warum sich die als junge Männer verkleideten Heldinnen sowohl in Trevor Nunns Verfilmung von $W$ as ihr wollt (1996) als auch in Shakespeare in Love zusätzlich einen falschen Schnurrbart ankleben, um den Sehgewohnheiten des 20. Jahrhunderts Rechnung zu tragen, denn die schönen Jünglinge bei Shakespeare sind gerade durch ihre Bartlosigkeit gekennzeichnet. $^{7}$

Dennoch ist Kleidungswechsel noch immer die einfachste Methode, Geschlecht zu dekonstruieren, da hierdurch deutlich gemacht wird, wie sehr wir uns in unserer Wahrnehmung von bestimmten gesellschaftlich abgesprochenen Zeichen leiten lassen, um nicht nur Geschlechtszugehörigkeit zu bestimmen, sondern, damit verbunden, auch Vermutungen über die Charaktereigenschaften unseres Gegenübers anzustellen. Cross-dressing bringt deshalb nicht nur die biologische Unterscheidung zwischen männlich und weiblich durcheinander, sondern ebenso die jener Verhaltensweisen, die angeblich mit dem Geschlecht eng verbunden sind. Seit den 1980er Jahren ist Transvestismus (cross-dressing) ${ }^{8}$ ein wachsendes

5 Man denke auch an die für unsere Sehgewohnheiten ,unmännliche` elisabethanische Herrenmode.

6 Ein Mann im Kleid steht jedoch noch immer unter Erklärungszwang.

7 So ruft etwa der Narr Feste in Was ibr wollt der verkleideten Viola zu: „Möge Gott, wenn er die nächste Lieferung Haare verteilt, dir einen Bart ablassen!“ (3.1.43-44) („Now Jove, in his next commodity of hair, send thee a beard!" 3.1.45-46) Die englischen Zitate aus Twelfth Night sind der von J. M. Lothian und T. W. Craik herausgegebenen Arden-Ausgabe entnommen. Die deutsche Übersetzung stammt von Frank Günther.

8 Der Begriff ,Transvestismus' bzw. ,Transvestitismus‘ (nicht zu verwechseln mit ,Transsexualität') hat im Laufe des letzten Jahrhunderts viele Bedeutungsänderungen erfahren (vgl. Garber 131ff.) und wird teilweise im Zusammenhang mit Fetischismus gebraucht, weswegen ich im Folgenden bevorzugt den neutraleren und mittlerweile auch im Deutschen üblichen englischen Terminus cross-dressing verwenden werde. 
interdisziplinäres Forschungsgebiet. Als besonders interessant hat sich dabei das England des späten 16. Jahrhunderts, die Zeit William Shakespeares, erwiesen.

Im elisabethanischen England gab es eine gesetzlich festgeschriebene Kleiderordnung, die vor allem darauf abzielte, die streng hierarchisch strukturierte gesellschaftliche Ordnung zu bewahren. ${ }^{9}$ Das Tragen von besonders wertvollen Stoffen und aufwendigen Kleidungsstücken etwa war dem Adel vorbehalten. Mode fungierte somit als äußeres Zeichen von Rang und Klassenzugehörigkeit, und ein Verstoß gegen die existierenden Regeln wurde als Vortäuschung falscher Tatsachen angesehen. Da Geschlecht in erster Linie eine soziale Kategorie war, wurden Frauen, die sich wie Männer kleideten, nicht etwa als sexuell abweichend im Sinne des 19. Jahrhunderts betrachtet. ${ }^{10}$ Der Skandal lag vor allem in der unerlaubten Aneignung von Privilegien, die ihnen als Frauen in einer streng patriarchalischen Gesellschaft nicht zustanden. Eindeutige Lesbarkeit von Zugehörigkeiten galt als unabdingbar für das Funktionieren des Soziallebens, und Gesetzesverstöße wurden mitunter hart bestraft. Aus Frankreich berichtet Montaigne gar von als Männer lebenden Frauen, die nach ihrer Entdeckung zum Tode verurteilt wurden.11 Solche Fälle sind, wie Stephen Greenblatt in seinem einflussreichen Aufsatz „Fiction and Friction" schreibt, als „Schattengeschichten“ (66) etwa in Was ibr wollt zwischen den Zeilen präsent: Was wäre passiert, wenn Violas Tarnung aufgeflogen wäre? Was, wenn Olivia und Cesario (also Viola) tatsächlich geheiratet hätten?

Angesichts dieser strengen disziplinarischen Maßnahmen und der Missbilligung jeder Art von Zweideutigkeit mag es verwundern, dass das Theater von diesen Regeln ausgenommen war. Wie mittlerweile weithin bekannt, wurden in England bis ins 17. Jahrhundert hinein alle Frauenrollen von Knaben gespielt, deren Stimmen noch ungebrochen waren. Scharfe Kritik an den boy actors formierte sich in England zuerst mit dem Aufstieg der Puritaner, deren polemische Schriften gegen die Schauspielerei trotz aller Hysterie heute als wertvolle historische Quellen für die elisabethanische Theaterpraxis dienen. Das Theater mit seinen verfälschenden Nachahmungen der Wirklichkeit war für die Puritaner prinzipiell Teufelszeug. In einem der zahlreichen Pamphlete etwa heißt es: „Wenn in Stücken ein Knabe die Kleidung, die Gestik und die Gefühle einer Frau annimmt, ein Armer den Titel eines Fürsten trägt, königliches Verhalten nachahmt und falsches Gefolge um sich schart, dann zeigen sich diese Personen äußerlich anders, als sie wirklich sind, und machen sich somit der Lüge schuldig." "Während sich hier das

9 Vgl. hierzu Garber 25ff und Jardine.

10 Howard weist darauf hin, dass Frauen, die sich wie Männer kleideten, oft für sexuell aggressiv bzw. für Huren gehalten wurden (100).

11 Den rechtlichen Umgang mit Fällen von (weiblichem) Transvestismus im England des 16. Jahrhunderts behandelt u.a. Howard 95ff..

12 „П] Stage Playes for a boy to put one the attyre, the gesture, the passions of a woman; for a meane person to take vpon him the title of a Prince with counterfeit porte, and traine, is by outwarde signes to shewe them selues otherwise then they are, and so with in the compasse of a lye." Stephen Gosson, Playes Confuted in fine Actions, 1582. Zit. in Sedinger 63. (Übers. der Verf.) 
Unbehagen noch ganz allgemein auf die Suggestion einer wandelbaren, von außen nicht eindeutig ablesbaren Identität bezieht, ganz gleich, ob Geschlechter- oder Standesgrenzen betreffend, standen bald vor allem die, falsch' gekleideten boy actors im Mittelpunkt der Anklage. Die Theaterpraxis stand ganz eindeutig im Widerspruch zu einer immer wieder zitierten biblischen Vorschrift: „Eine Frau soll nicht Männersachen tragen, und ein Mann soll nicht Frauenkleider anziehen; denn wer das tut, der ist dem HERRN, deinem Gott, ein Gräuel.“ (5. Mose, 22,5) Was die Puritaner neben der Verletzung eines göttlichen Gebots verstörte, war die Gefahr eines Verwischen von Geschlechterdifferenz, und das Entfachen von Lust in all ihren Formen bei den männlichen Zuschauern. Über die Wirkung auf das ebenfalls stets stark vertretene weibliche Publikum machte man sich anscheinend keine Gedanken, und auch die Literaturwissenschaft hat sich erst spät für den weiblichen Blick auf die verkleideten Körper interessiert. ${ }^{13}$

Natürlich hatten die Puritaner nicht ganz unrecht, wenn sie von der Auflösung der Geschlechtergrenzen sprachen. In einer Schrift von 1583 heißt es: „Unsere Kleidung wurde uns [von Gott] als festes Zeichen dafür gegeben, zwischen den beiden Geschlechtern unterscheiden zu können. Wer deshalb die Kleidung des anderen Geschlechts trägt, hat Anteil an diesem, und verfälscht so die Wahrheit seines eigenen. " 14 Auch wenn diese ,Verfälschung' im vom Alltag abgetrennten Raum des Theaters stattfand und das Publikum in jedem Fall eingeweiht war, also um die biologische Männlichkeit der Frauendarsteller wusste, mag hier etwas von der transgressiven Wirkung der boy actors aufscheinen und erklären, warum Shakespeare in mehreren seiner Komödien Kleidertausch als Handlungselement einsetzt. Explizit thematisiert wird das standardmäßige cross-dressing auf der Bühne nämlich nur dann, wenn das Annehmen einer fremden Geschlechterrolle auch Teil des Plots ist. Eine Ausnahme ist hier die oft in diesem Zusammenhang zitierte Stelle aus Shakespeares Antonius und Kleopatra, in der sich Kleopatra kurz vor ihrem Selbstmord ausmalt, wie ihre tragische Geschichte in zukünftigen Theateraufführungen der Lächerlichkeit preisgegeben wird:

\section{Marc Anton}

Wird reingeschleppt im Suff, und ich seh meine Hoheit

Hingekleopatrat von einem Quäkstimm-Knaben, Hohnvoll in Hurenposen. ${ }^{15}$ (5.2.217-20)

Auf diese erst spät gefüllte Forschungslücke verweist u.a. Sedinger (66).

„Our Apparell was given us as a signe distinctive to discern betwixt sex and sex, and therefore one to weare the Apparel of another sex, is to participate with the same, and to adulterate the verities of his owne kinde." Philip Stubbes, The Anatomy of Abuses. 1583. Zit. in Howard 97. (Übers. der Verf.)

„Antony / Shall be brought drunken forth; and I shall see / Some squeaking Cleopatra boy my greatness / I'th' posture of a whore.“ (5.2.217-220) Die englische Fassung folgt der von John Wilders herausgegebenen Arden-Ausgabe. Die deutsche Übersetzung stammt von Frank Günther. 
Der selbstreferentielle Verweis auf den Knabenschauspieler, der diese Zeilen spricht, birgt natürlich - vor allem für unsere Sehgewohnheiten - die Gefahr eines Illusionsbruchs, den Shakespeare anscheinend zu riskieren bereit war. ${ }^{16}$

Noch in der Mitte des 17. Jahrhunderts wurden Schauspieler wie Edward Kynaston als überzeugendere und - wie beispielsweise die Tagebuchaufzeichnungen von Samuel Pepys bezeugen - schönere Bühnenfrauen gefeiert. ${ }^{17}$ Auch wenn die Ablösung der boy actors durch Schauspielerinnen in der Restaurationszeit als Schritt auf dem Weg zur Gleichberechtigung der Frauen zu sehen ist, sollte man nicht zu schnell der Vorstellung erliegen, nur eine ,echte' Frau könne auf der Bühne glaubhaft weibliches Verhalten darstellen. Gleiches gilt natürlich auch für die Darstellung von Männlichkeit: Sarah Bernhardt und viele andere Schauspielerinnen haben Hamlet als Hosenrolle (also als Mann) gespielt, ${ }^{18}$ und Cate Blanchett wurde von Regisseur Todd Haynes in seinen Film I'm Not There (2007) als Bob Dylan besetzt. Sicher, das Theater der Renaissance und der Restauration war kein in unserem Sinne naturalistisches Theater, sondern lebte von seiner betonten Künstlichkeit. Frauen- wie Männerdarstellungen basierten auf bestimmten Gesten, Körperhaltungen und antrainierten Sprechweisen. ${ }^{19}$ Gleichzeitig sollte man sich jedoch klarmachen, dass alles, was auf einer Bühne passiert, immer bestimmten Konventionen unterworfen ist, und zwar nicht nur solchen, die das Theater betreffen. Denn was ist denn für den Zuschauer eine glaubhafte Imitation einer ,echten ${ }^{6}$ Frau oder eines ,echten` Mannes? Hier sind wir wieder beim alten Problem der Normierung von Genderrollen. Hilft uns nicht vielleicht die Artifizialität der elisabethanischen Bühne dabei, die alltäglich zu beobachtenden gender performances viel genauer in den Blick zu bekommen beziehungsweise sie als solche überhaupt erst zu erkennen? Auf der Bühne muss man immer spielen. Es reicht nicht, einfach

16 Zur Problematik des boy actors in der Renaissance vgl. Stallybrass, der darauf hinweist, wie wenig wir letztlich über die Theaterpraxis der Shakespearezeit wissen. Die vielen Entkleidungsszenen, in denen ganz deutlich der weibliche Körper in den Blick gerückt wird (beispielsweise in Othello und Antonius und Kleopatra, wo sich Kleopatra im letzten Akt eine Giftschlange an den Busen hält), werfen etwa die Frage auf, ob die Knabenschauspieler eventuell künstliche Brüste getragen haben. Von Interesse ist in diesem Zusammenhang außerdem die Studie von Stephen Orgel.

17 Am 18. August 1660 notierte Pepys: „Ein gewisser Kynaston, ein Knabe, spielte die Schwester des Herzogs und verkörperte die entzückendste Dame, die ich jemals in meinem Leben gesehen habe.“ (,[O]ne Kynaston, a boy, acted the Duke's sister but made the loveliest lady that ever I saw in my life.") Zit. in Orgel 33. (Übers. der Verf.)

Ein Beispiel wäre Angela Winkler in Peter Zadeks 1999er Inszenierung für die Wiener Festwochen. Asta Nielsen, wohl eine der berühmtesten Hamlet-Darstellerinnen, bildet hier streng genommen eine Ausnahme, da der Stummfilm von 1920 den schwarzen Prinzen als Frau in Männerkleidern präsentiert. Der Film macht dennoch interssante Aussagen zum Thema Gender als performance. Zu Hamlet als Hosenrolle vgl. Howards.

19 Einen Eindruck davon, wie diese Darstellungskonventionen ausgesehen haben könnten, vermittelt Richard Eyres Film Stage Beauty (nach dem Stück Compleat Female Stage Beanty von Jeffrey Hatcher), der vom Ende der Karriere Edward Kynastons als Frauendarsteller erzählt. 
eine Frau oder ein Mann zu sein. Und dass die Welt ebenfalls eine Bühne ist, darauf verweist uns der Melancholiker Jacques in Wie es euch gefällt.

Die 1991 er Aufführung von Wie es euch gefällt durch die Theatergruppe Cheek by Jowl, in der alle Rollen mit (allerdings durchwegs erwachsenen) Männern besetzt wurden, war für viele Zuschauer ein Aha-Erlebnis. Wenn auch nicht zu vergleichen mit den Knabenschauspielern des 16. und 17. Jahrhunderts, wurde hier bewiesen, dass ,Weiblichkeit' überzeugend von Männern dargestellt werden kann, ohne gleich camp zu wirken. Adrian Lester, der die Rolle der Rosalinde spielte, berichtete später, dass er auf das Publikum immer dann einen besonders weiblichen Eindruck machte, wenn sich Rosalinde-als-Ganymed bemühte, macho zu wirken. ${ }^{20}$

Rosalinde ist die wortgewandte Tochter des von seinem Bruder verdrängten Herzogs, der mit seinen Gefolgsleuten im Ardenner Wald Zuflucht gefunden hat und dort das pastorale Leben genießt. Bald muss sie ebenfalls vom Hofe fliehen und macht sich gemeinsam mit ihrer Kusine Celia in den Wald auf. Zum Selbstschutz nimmt Rosalinde die Rolle eines Schäferjungen an und nennt sich ab sofort Ganymed. Dass Rosalinde von allen ihr zur Verfügung stehenden Männernamen ausgerechnet diesen wählt, verdient durchaus Beachtung. ${ }^{21}$ In der griechischen Mythologie, mit der Shakespeares gebildete Zeitgenossen gut vertraut waren, war Ganymed ein hübscher Hirtenknabe, den Zeus in Gestalt eines Adlers entführte, um ihn im Olymp zu seinem Liebhaber und zum Mundschenk der Götter zu machen. Ganymede und die über die lateinische Version des Namens abgeleitete Form catamite waren außerdem im elisabethanischen Slang gängige Begriffe für männliche Prostituierte. ${ }^{22}$ Rosalindes Namenswahl situiert das Stück also im Kontext pastoraler Homoerotik, wie man sie von Vergil kannte. ${ }^{23}$

Als Ganymed trifft Rosalinde den in sie verliebten Orlando wieder, der sie in ihrer Verkleidung nicht erkennt. Ganymed verspricht Orlando, ihn von seiner Liebeskrankheit zu heilen, wenn er ihn mit Rosalinde anreden und um ihn werben wolle. Die Sache wird nun einigermaßen kompliziert: Rosalinde spielt Ganymed, der nun wiederum eine fiktive Rosalinde spielt. Und natürlich wird die Bühnenfigur von einem Knaben gespielt. Die erfundene Rosalinde hat mit der ,echten Rosalinde, wie wir sie zu Beginn des Stücks kennengelernt haben, nichts zu tun. Sie ist eine Klischeefigur, die sich aus literarischen Vorbildern wie der unerreichbaren Dame aus der petrarkistischen Liebesdichtung und stereotypen Vorstellungen von weiblichem Wankelmut zusammensetzt. Diese Rosalinde quengelt, ist

20 Die Einleitung von Juliet Dusinberre in der aktuellen Arden-Ausgabe von As You Like It enthält das oben paraphrasierte Zitat von Lester sowie ein Foto des Schauspieleres in seinem Rosalinde-als-Ganymed-Kostüm (20-21).

21 Orgel (43) weist darauf hin, dass die homoerotischen Assoziationen, die mit dem Namen Ganymed verbunden sind, von den meisten Kritikern und Kommentatoren lange ignoriert wurden.

22 Vgl. hierzu Franceschina 3 und 19ff.

23 Zur Rezeption von Vergils Eklogen in der englischen Literatur des 16. Jahrhunderts vgl. Smith 79-115. 
abwechselnd herrisch und weinerlich - sie ist die Manifestation einer misogynen Fiktion. Rosalinde ist also auch als Rosalinde II nicht einfach Frau, sondern sie spielt Frau und weist so auf die grundlegende Künstlichkeit von Genderrollen hin. Im Laufe des raffinierten Rollenspiels, das auf Rosalindes Initiative hin begonnen wurde, weichen die Unterschiede zwischen Ganymed und Rosalinde immer mehr auf. Schließlich kommt es sogar zu einer gespielten Hochzeit zwischen Ganymedals-Rosalinde und Orlando, der die Zeremonie auffallend ernst zu nehmen scheint. Die homoerotischen Momente, die sich zwischen Ganymed und Orlando entwickeln, werden durch eine weitere Liebesgeschichte ergänzt, in die Rosalinde verwickelt ist: Die Schäferin Phoebe verliebt sich in den hübschen Schäferjungen Ganymed und scheint gerade von der Mischung aus weiblichem Aussehen und arroganter männlicher Haltung angezogen zu werden:

Es ist ein hübscher Kerl - nicht allzu hübsch -,

Doch sicher stolz - doch steht sein Stolz ihm gut.

Das wird ein strammer Mann. Das Beste dran

Ist sein Gesicht; und schneller als sein Mund

Verletzen kann, heilt es sein Auge zu.

Sein Bein ist nur soso - und trotzdem gut.

Es war ein schönes Rot auf seinen Lippen,

Ein Rot, ein bisschen feuriger und reifer

Als auf den Wangen; grad der Unterschied

Von Karmesin- zu Purpurrosen war's.

$$
(3.5 .113-23)^{24}
$$

Dem traditionellen Schönheitskatalog scheint Ganymed nicht zu entsprechen. Doch je mehr Phoebe versucht, kein gutes Haar an ihm zu lassen, desto schwärmerischer wird sie.

Es ist vielleicht bezeichnend für die Zeit um 1800, dass unter den für John Boydells Shakespeare-Galerie ausgewählten Szenen gerade jene zu finden ist, in der Ganymed ,typisch weibliches' Verhalten an den Tag legt: Als er/sie die Nachricht von Orlandos Verletzung in Form eines blutgetränkten Taschentuchs erreicht, sinkt Ganymed in Ohnmacht, auch wenn er später darauf besteht, diese nur gespielt zu haben.

Am Ende des Stücks ist der Ausflug in die pastorale Wunderwelt mit ihren erotischen Verwicklungen zu Ende. Der Herzog und sein Gefolge kehren aus dem

24 „It is a pretty youth - not very pretty - / But sure he's proud, and yet his pride becomes him. / He'll make a proper man. The best thing in him / Is his complexion; and faster than his tongue / Did make offence, his eye did heal it up. / He is not very tall, yet for his years he's tall; / His leg is but so-so; and yet 'tis well. / There was a pretty redness in his lip, / A little riper and more lusty red / Than that mixed in his cheek. 'Twas just the difference / Betwixt the constant red and mingled damask." (3.5.114-124) Die englischen Zitate aus As You Like It folgen dem Text der von Juliet Dusinberre herausgegebenen Arden-Edition. Die deutsche Übersetzung stammt von Frank Günther. 
Exil an den Hof zurück, und Rosalinde trägt wieder Frauenkleider - ein Hochzeitskleid, um genau zu sein, denn zuletzt stehen gleich vier zu vermählende Paare auf der Bühne. ${ }^{25}$ Mit dem Ablegen ihres Schäferkostüms und damit der Rolle des Ganymed nimmt Rosalinde wieder den ihr zugewiesenen Platz in der gesellschaftlichen Hierarchie ein und wird von ihrem Vater Orlando zugeführt. Hymen, der Gott der Ehe, verkündet etwas unwirsch: „Still! Die Verwirrung end ich, / Die Wunderdinge wend ich / Zum Schluss, der alles eint"“ (5.4.121-23). ${ }^{26}$

Dieses angekündigte Ende aller Verwirrungen und die durch den Kostümwechsel auch visuell kenntlich gemachte Rückkehr in geordnete Bahnen werden jedoch durch den an das Stück anschließenden Epilog, für den Rosalinde noch einmal in ihrem Hochzeitskleid auf die Bühne kommt, um das Publikum direkt anzusprechen, erneut in Frage gestellt.

Die Heldin als Epilog, das ist ganz gegen Brauch und Sitte; aber auch nicht alberner, als wenn der Held den Prolog spricht. [...] Wenn ich eine Frau wäre, ich würde jeden von euch küssen, der einen Bart hat, der mir gefällt, ein Aussehn, das mir zusagt, und einen Atem, der mich nicht abstößt. Und ich bin sicher, alle, die ihr hübsche Bärte, hübsche Gesichter und reinen Atem habt, werdet mir, wenn ich meinen Knicks mache, für mein liebenswürdiges Angebot ein herzliches ,Auf bald!' sagen.

$$
\text { (Epilog, 1-21) }
$$

It is not the fashion to see the lady the epilogue; but it is no more unhandsome than to see the lord the prologue. [...] If I were a woman, I would kiss as many of you as had beards that pleased me, complexions that liked me, and breaths that I defied not. And I am sure, as many as have good beards, or good faces, or sweet breaths, will for my kind offer, when I make curtsy, bid me farewell.

Im Epilog zieht plötzlich der Schauspieler die Aufmerksamkeit auf sich, der ganz klar zwischen dem Geschlecht der Figur, die er gerade gespielt hat, und seinem eigenen unterscheidet. Die dramatische Illusion, dass es sich bei Rosalinde um ein gerade verheiratetes Mädchen handelt, wird radikal durchbrochen, wenn der boy actor mit den Männern im Publikum flirtet. Wer ist es aber nun tatsächlich, der hier spricht? Einerseits ist es Rosalinde, die Heldin des Stücks, andererseits der Schauspieler, der seinen Applaus einfordert. Und irgendwie ist es vielleicht auch der flüchtige Ganymed, der sich hier in einer weiteren doppelgeschlechtlichen

25 Aufgrund diverser editorischer Unklarheiten bleibt die Frage, ob Rosalinde in dieser Szene ihr Knabenkostüm tatsächlich abgelegt hat, allerdings letztlich offen. Vgl. zu dieser Kontroverse den Aufsatz von Masten sowie Orgel 33.

26 „Peace ho! I bar confusion. / 'Tis I must make conclusion / Of these most strange events.“ (5.4.123-25) 
Inkarnation noch einmal zu Wort meldet. Marjorie Garber vergleicht die nicht wirklich greifbare Präsenz jener quasi-virtuellen Figur namens Ganymed mit dem Lächeln der Grinse-Katze aus Alice im Wunderland, das körperlos in der Luft schwebt, nachdem seine Besitzerin bereits verschwunden ist (75).

Auch das um 1600, kurz nach Wie es euch gefällt verfasste Was ibr wollt spielt in einem vom Alltagsleben abgetrennten Raum, im Land Illyrien, das zwar einerseits Ähnlichkeiten mit England aufweist und außerdem geographisch im Mittelmeerraum verortet werden kann, aber insgesamt deutlich als eine Art romanzenhaftes Paralleluniversum konstruiert ist. Dementsprechend fantastisch sind auch die Zufälle und ungeheuerlichen Begebenheiten, die das Stück selbst als theatralische Tricks identifiziert. Gegen Ende des dritten Aktes kommentiert der Diener Fabian: „Wenn man so was auf der Bühne darstellt, würd ich fragen, was hat das mit der Wirklichkeit zu tun?" (3.4.123-24) ${ }^{27}$

Der Feiertag, nach dem das Stück in der englischen Fassung benannt ist, Twelfth Night, ist der 6. Januar, das Dreikönigsfest und die 12. Nacht der Weihnachtsfestlichkeiten. Dieser Tag stand ganz im Zeichen einer karnevalesken verkehrten Welt, an dem sonst bestehende Hierarchien auf den Kopf gestellt werden. Der Nebentitel, What You Will, ebenso allumfassend und auf die Unterhaltungsfunktion des Stückes hinweisend wie As You Like It, mag außerdem ein Wortspiel mit Shakespeares Vornamen enthalten. Das Wort will konnte im 16. Jahrhundert zudem alle möglichen obszönen Bedeutungen haben, und würde somit bereits auf die diversen erotischen Verstrickungen hinweisen, die die Zuschauer erwarten.

$\mathrm{Zu}$ den Vorlagen des Dramas zählen neben einem zeitgenössischen italienischen Stück Shakespeares eigene frühe Komödie der Irrungen (Comedy of Errors), die ihrerseits einen Versuch darstellt, die dramatischen Möglichkeiten der Menaechmi des Plautus zu überbieten. ${ }^{28}$ Wo im antiken Vorbild ein Zwillingspaar für Verwechslungen am laufenden Band sorgt, sind es bei Shakespeare gleich zwei, jeweils Herr und Diener. In $W$ as ibr wollt stehen nun zweigeschlechtliche Zwillinge im Mittelpunkt der Handlung, wodurch sich das neue Motiv des Geschlechtertauschs anbietet, denn die beiden sind nicht auseinanderzuhalten, wenn sie die gleiche Kleidung tragen. Die Heldin, Viola, ist erfolgreich im Nachahmen männlichen Verhaltens, weil sie ihre performance an ihren tot geglaubten Zwillingsbruder Sebastian anlehnt. ${ }^{29}$ Wie Rosalinde als Ganymed ist Viola als Page Cesario überaus eloquent und schlägt sich brillant in den Wortgefechten mit dem Narren Feste. In ihrer Rolle als übermütiger bis unverschämter Junge scheint sie voll aufzugehen.

Allerdings ist ihre Lage weitaus heikler als die von Rosalinde, befindet sich Viola doch nicht in einer pastoralen Waldidylle, sondern an einem Fürstenhof. Die

27 „If this were played upon a stage now, I could / condemn it as an improbable fiction.“ (3.4.12829)

28 Zu Shakespeares Quellen vgl. die Einleitung der Arden-Ausgabe.

29 In der Filmfassung von Trevor Nunn sieht der Zuschauer Viola und Sebastian vor dem Schiffbruch als Darsteller in einem drag act. 
Angst vor Entdeckung und das Fehlen einer Vertrauensperson machen Violas Situation immer schwieriger. Während Rosalinde ihre Verkleidung auch ohne äußerlichen Grund beibehält und nur deshalb Ganymed bleibt, weil sie Spaß an dem Rollenspiel mit Orlando gefunden hat und ihren Part als Phoebes unwilliger Liebhaber zu genießen scheint, gerät Viola, als sie in ein Duell verstrickt wird, sogar in eine potentiell lebensbedrohliche Situation.

Interessanterweise erfährt der Theaterzuschauer erst in der letzten Szene den Namen der Heldin, nämlich dann, als ihr Zwillingsbruder Sebastian wieder auftaucht, sie mit ,Viola' anspricht und ihr so auf fast magische Weise mit ihrem Namen auch ihre alte Identität zurückgibt. Die verliebte Olivia hat inzwischen den an seinem Verstande zweifelnden Sebastian geheiratet, in der Annahme, er sei Cesario, und Orsino macht Cesario, kaum dass dessen Geschlecht bekannt gemacht wurde, ebenfalls einen Heiratsantrag.

Junge, du hast mir tausendmal gesagt,

Du würdest keine Frau je lieben so wie mich.

[...] Deine Hand,

Und zeig dich mir in deinen Mädchenkleidern. $(5.1 .261-68)^{30}$

Interessanterweise wird Viola von Orsino auch weiterhin als boy bzw. als Cesario angesprochen, solange sie noch in Hosen steckt. Vielleicht ist Orsino selbst ein bisschen erschrocken über seine schnelle Heiratswilligkeit - schließlich hat er gerade erst erfahren, dass es sich bei seinem Lieblingspagen um eine Frau handelt - und er vertagt die Anerkennung Violas als Geliebte in die Zukunft, wenn sie ihm in Frauenkleidern gegenübersteht. Diese Lesart wirft allerdings die Frage auf, warum uns das Stück um den Anblick von Viola in ihren ,echten'Kleidern bringt und uns einen fadenscheinigen Grund für deren komplizierte Wiederbeschaffung auftischt. Rosalinde erscheint in der letzten Szene in ihrem Hochzeitskleid und gibt sich Orlando als Frau zu erkennen. Mit dieser Rückverwandlung, bewirkt durch einen Kleiderwechsel, wird die Geschlechterverwirrung innerhalb der Handlung zumindest optisch aufgehoben, auch wenn der Epilog hier einiges wieder ins Schwanken bringt. Viola bleibt für Orsino und für die Zuschauer bis zum Schlusstableau Cesario, die Kunstfigur, in die sich Orsino und Olivia verliebt haben. Auch Sebastian, der gerade im rechten Moment an Olivias Hof kommt, um von ihr geheiratet zu werden, löst in seiner an sie gerichteten Rede die Geschlechterambivalenzen durch ein Wortspiel nicht völlig auf:

Fräulein, so kam's zustand. Sie irrten sich.

Doch kam Natur mit Bandespiel noch zu sich selbst.

Sie wollten eine junge Frau zum Mann -

Und solln sie haben; denn verlobt sind Sie

30 „Boy, thou hast said to me a thousand times / Thou never should'st love woman like to me. / [...] Give me thy hand, / And let me see thee in thy woman's weeds.“ (5.1.265-271) 
mit einer Jung-frau auch in Ihrem Mann. (5.1.253-57) ${ }^{31}$

Eine ähnliche fast alchemistische Verbindung eines männlichen und eines weiblichen Begriffs findet sich parallel zu dieser Stelle auch in Bezug auf Viola/Cesario, wenn Orsino verkündet:

Und weil Sie ,Herr' so lange zu mir sagten,

Hier meine Hand; Sie solln von heute an

Des Herren Herrin sein. (5.1.316-318) ${ }^{32}$

Hier lässt sich außerdem ein intertextuelles Echo erkennen. Shakespeares berühmt-berüchtigtes Sonett Nr. 20, das zu den an einen jungen Mann adressierten Gedichten gehört und dessen androgyne Schönheit preist, beginnt mit den folgenden Zeilen:

Ein frauenantlitz das Natur selbsthändig

Gemalt - hast du Herr-Herrin meiner minne 33

Ich werde nun einen Exkurs in das 19. Jahrhundert unternehmen und eine der interessantesten Adaptionen von Wie es euch gefällt in den Blick nehmen. Théophile Gautiers erotischer Roman Mademoiselle de Maupin aus dem Jahre 1835 gehört zu den Vorläufertexten des Ästhetizismus. Bekannt wurde er vor allem aufgrund seines polemischen Vorworts, das als Manifest der l'art pour l'art-Idee die Kunst vor moralisierenden Kritikern in Schutz nimmt. Doch auch die Beschäftigung mit nicht-normativen Formen des Begehrens und die zentrale Funktion, die Maskerade und Rollenspiel innerhalb der Romanhandlung einnehmen, wirkte prägend für Autoren wie Oscar Wilde oder Künstler wie Aubrey Beardsley, der mehrere Illustrationen zu Gautiers Roman schuf.

Mademoiselle de Maupin handelt von der Suche des jungen Chevalier d'Albert nach seiner Traumfrau, die er sich aus literarischen Vorbildern und Gemälden antiker Göttinnen zusammengebastelt hat. Als echter Romantiker verzehrt er sich also nach einem fantastischen Hirngespinst, dessen tatsächliche Existenz getrost bezweifelt werden darf. Als seine Affäre mit der hübschen Rosette - für ihn ohnehin nur eine Übergangslösung - immer unbefriedigender wird, sorgt ein neuer Gast auf dem Château für Aufregung und Erregung: Sowohl d'Albert als auch Rosette verlieben sich in den androgynen Schwertkämpfer Théodore de Sévanne.

31 „So comes it, lady, you have been mistook. / But nature to her bias drew in that. / You would have been contracted to a maid; / Nor are you therein, by my life, deceiv'd: / You are betroth'd both to a maid and man." (5.1.257-261) „And since you call'd me master for so long, / Here is my hand; you shall from this time be / Your master's mistress.“ (5.1.323-25)

33 Die Parallele ist in der englischen Originalfassung noch deutlicher: „A woman's face with nature's own hand painted / Hast thou, the master-mistress of my passion." Die deutsche Übersetzung stammt von Stefan George und findet sich in der zweisprachigen Reclam-Ausgabe von Shakespeares Sonetten. 
D'Albert ist sich der Ironie durchaus bewusst, dass die in seinen Augen perfekte Frau in der Gestalt eines jungen Mannes daherkommt, und er hält das Ganze für einen göttlichen Betriebsfehler. Denn warum sollte solche Schönheit an einen bloßen Mann verschwendet werden? In einem Brief gesteht d'Albert seinem Freund Silvio: „[A]lles ist mir umgestürzt und in tiefster Verwirrung; ich weiß kaum mehr, wer ich bin, noch wer die anderen sind, weiß nicht, ob ich Frau oder Mann bin, [...] in manchen Augenblicken will mir scheinen, als verlöre ich den Verstand [...]. Silvio, ich liebe ... wie schwer wird mir, es Dir zu sagen ... ich liebe einen Mann!“" (Gautier 169-70) Erst nach diesem erstaunlichen und in der Literatur bis dahin einzigartigen Comingout ${ }^{34}$ wird dem bereits ahnungsvollen Leser enthüllt, dass sich hinter der Persona des schönen Théodore die Titelheldin, Madeleine de Maupin, verbirgt. Diese befindet sich quasi im Undercovereinsatz. In dem Wunsch, die Männer besser kennenlernen, bevor sie sich an einen von ihnen bindet, hat sie beschlossen, einer zu werden und Feldforschung am lebenden Objekt zu betreiben. Ähnlich wie Viola in Shakespeares Was ibr wollt gelingt es ihr dabei, viel näher an ihren zukünftigen Liebhaber heranzukommen, als ihr das als Frau jemals möglich gewesen wäre. Im Gegensatz zu Viola, die sich schnell in Orsino verliebt, bleibt dieser weibliche Spion jedoch zunächst unbeeindruckt von d'Alberts Charme und fühlt sich im übrigen von der frauenfeindlichen Haltung der meisten Männer zutiefst abgestoßen.

Die Nähe zu Shakespeares Komödien wird explizit gemacht, als die versammelten Schlossgäste sich vornehmen, gemeinsam Wie es euch gefällt aufzuführen. Die Auswahl des Stückes ist natürlich kein Zufall. Ganz bewusst bedient sich Gautier der Shakespeareschen Komödie, um die komplexen Begehrensstrukturen und Identitätskrisen zu erforschen, kurz, etwas, was wir heute als queer bezeichnen würden. Da sich Rosette weigert, Männerkleider zu tragen, übernimmt Théodore (also Madeleine) die Rolle der Rosalinde, und Rosette die der Schäferin Phoebe, die sich in die als Jüngling verkleidete Rosalinde verliebt. Beim Versuch, den Drehungen der Schraube noch folgen zu wollen, kann einem schon schwindelig werden: Madeleine de Maupin ist eine Frau, die einen Mann spielt, der eine Frau spielt, die einen Mann spielt, der eine Frau spielt. Irgendwo in der dritten, spätestens in der vierten Umdrehung mag einen die Vermutung beschleichen, dass die Kategorien ,Mann“ und ,Frau' unbrauchbar geworden sind, um zu beschreiben, was hier tatsächlich geschieht. Auch d'Albert, der sich zunächst sehr gegen seine (vermeintlich) homosexuellen Gefühle zu sträuben versucht, erahnt bald, dass biologisches Geschlecht womöglich irrelevant ist, wenn erotisches Begehren erst einmal geweckt wurde.

Seltsam ist, dass ich fast nicht mehr an sein Geschlecht denke und ihn liebe in vollkommener Sicherheit. Zuweilen noch suche ich mich davon zu überzeugen, dass diese Neigung abscheulich ist, und gehe streng mit mir ins Ge-

$34 \quad$ Vgl. Duncker xix. 
richt. Aber nur äußerlich ist dies Beginnen, es entstammt dem Verstand und keineswegs dem Gefühl. In Wirklichkeit scheint mir alles einfach und selbstverständlich, und ich rede mir ein, jeder andere würde an meiner Stelle ebenso tun. (190)

Madeleine gerät ebenfalls an einen Punkt, an dem sie die Trennung der Geschlechter in Frauen und Männer und die damit einhergehenden Vorschriften für die Partnerwahl als einengend empfindet. Als die verliebte Rosette sich daran macht, sie zu verführen, denkt sie, „Der Gedanke an eine Geschlechtsgleichheit verblasste allmählich, und nach und nach blieb nur noch ungewiss lustvolle Vorstellung" (299).

Auf bemerkenswert moderne Weise zeigt Gautiers Roman, wie mangelhaft die Kategorien männlich und weiblich zu sein scheinen, sobald es darum geht, erotisches Begehren und sexuelle Identität definieren zu wollen. Madeleine de Maupin sehnt sich in keiner Weise zurück nach ihren Frauenkleidern. Sie genießt es, ihre aggressive, draufgängerische Seite ausleben zu können und sich frei in der Welt zu bewegen - Dinge, die gesellschaftlich und kulturell als männlich gelten, aber nichtsdestoweniger Teil ihrer Persönlichkeit sind, also rein gar nichts mit dem biologischen Geschlecht zu tun haben. Im Gegensatz zu Viola, die sich als „poor monster", also als armes Monstrum, beschreibt und sich zunehmend unwohler in ihrer Verkleidung fühlt, ist sich Madeleine bewusst, dass sie weder Mann noch Frau ist, sondern etwas ganz Neues. „Ich gehöre einem dritten, gesonderten Geschlechte an, das noch keinen Namen hat" (358). Und sie befürchtet, sollte sie jemals wieder ihre Frauenkleider herauskramen, würde sie wie ein verkleideter Mann wirken.

Die Identifikation der Romanfiguren mit den Rollen, die sie in Wie es euch gefällt spielen, geht so weit, dass sie schließlich auch deren Namen übernehmen, und somit die Handlung des Stücks im Alltag fortzuschreiben versuchen. Als sich d'Albert, der Orlando spielt, endlich dazu durchringt, einen Liebesbrief an Théodore zu schreiben, spricht er ihn mit Rosalinde an, und hält sich somit an die Aufgabe, die Shakespeares Rosalinde ihrem liebeskranken Orlando abverlangt: „Sie sind heilbar, wenn Sie mich nur Rosalinde nennen und jeden Tag zu meiner Hütte kommen und mir den Hof machen" (3.2.401). ${ }^{35}$ Und in ihrem Rosalinde-Kostüm verführt Madeleine schließlich ihren Orlando-d'Albert. Doch Gautier belässt es nicht dabei. Zwar sieht sich d'Albert als, natürlicher' Gewinner des Liebeswettstreits, als Madeleine sich ihm als Frau enthüllt, und er geht davon aus, dass Rosettes Verlangen nach Théodore - wie das von Phoebe nach Ganymed - unbefriedigt bleiben muss, doch im Roman, anders als bei Shakespeare, bekommt Phoebe zuletzt, was sie will. „Ich würde dich lieben, wenn ich könnte“ (5.2.104), ${ }^{36}$ sagt

35 „I would cure you, if you would but call me Rosalind, and come every day to my cote and woo me." (3.2.408-09)

36 „I would love you if I could.“ (5.2.107) 
Ganymed im Stück zu Phoebe und verspricht ihr, sollte er jemals eine Frau lieben, würde seine Wahl auf sie fallen. Diese unmögliche Liebe wird im Roman möglich gemacht, denn Madeleine verlässt ihren erschöpften Liebhaber, um sich dann - als Rosalinde - in Rosettes Schlafzimmer zu schleichen, das sie am nächsten Morgen als Théodore verlässt. Obwol der Roman nicht mit erotischen Szenen zwischen den beiden Frauen geizt, wird die Liebesnacht der beiden Frauen nicht beschrieben, sondern der Phantasie der Leser überlassen. Das doppelgeschlechtliche Wesen Théodore-Madeleine behält somit etwas von seinem Geheimnis, und es verwundert nicht, dass die schöne Chimäre am nächsten Morgen verschwunden ist. In einem zurückgelassenen Brief fordert sie d'Albert und Rosette auf: „Liebet Euch innig in Erinnerung an mich, die Ihr einer wie der andere geliebt, und sprecht zuweilen meinen Namen in einem Kuss!“ (Gautier 381) Mit diesem Satz endet der Roman und Patricia Duncker (xxviii) fragt zurecht, welchen Namen die Liebenden wohl im Munde führen werden - Madeleine, Théodore, Rosalinde oder Ganymed?

Eine aktuellere Auseinandersetzung mit der Shakespeareschen Komödientradition bietet Shakespeare in Love. John Maddens Film aus dem Jahre 1998, für den Marc Norman und der englische Dramatiker Tom Stoppard das Drehbuch schrieben, war ein oscargekrönter Publikumserfolg. Mit viel Einfallsreichtum und zahlreichen Verweisen auf diverse Shakespeare-Texte stellt die romantische Komödie Spekulationen über die Entstehungsgeschichte von Romeo und Julia an. Da unser Wissen über Shakespeares Leben sehr lückenhaft ist, gibt es genug fehlende Puzzlestücke, um hier interessante und unterhaltsame Thesen aufzustellen. Paradoxerweise konstruiert der Film jedoch trotz der vielen Insider-Witze, die sich an informierte Fans richten, ein Shakespeare-Bild, das sich eher an der Bardolatrie des 19. Jahrhunderts und den damit verbundenen Vorstellungen von Autorschaft und Identität orientiert als an den Erkenntnissen der letzten fünfzig Jahre. Der Film geht von der romantischen Idee aus, nur das selbst Erlebte könne in hohe Literatur umgewandelt werden. (Mit Blick auf Shakespeares Tragödien übrigens eine einigermaßen verstörende Vorstellung.) Man weiß jedoch, dass so gut wie alle Handlungsverläufe von Shakespeares Dramen, auch der von Romeo und Julia, auf bereits existierenden Vorlagen beruhen. ${ }^{37}$ Die tag line auf dem Filmplakat, „Love is the only inspiration“ („Liebe ist die einzige Inspiration“), ist also als These kaum haltbar, denn dass weder Romeo und Julia noch Shakespeares Sonette tagebuchartige Dokumente einer Liebesaffäre mit einer schönen blondgelockten Frau sind, ${ }^{38}$ gehört zu den wenigen Dingen, die wir tatsächlich über Shakespeare wissen. ${ }^{39}$

37 Eine Ausnahme ist Shakespeares letztes Drama Der Sturm (The Tempest).

38 Die 154 Sonette sind fast alle an einen (unbekannten) blonden jungen Mann gerichtet; die letzten 28 beschreiben eine selbstquälerische Affäre mit einer schwarzhaarigen femme fatale.

39 Vgl. Stephen Greenblatts Kommentar zum Film Shakespeare in Love „About that Romantic Sonnet" in der New York Times vom 6. Februar 1999. 
Die Handlung des Films ist schnell erzählt: Der junge Will Shakespeare leidet an writer's block. Da sich dieser fiktive Shakespeare die Grundgerüste seiner Stücke nicht aus fremden Texten zusammenbastelt, muss auf andere Weise Abhilfe für den Ideenmangel geschaffen werden - eine Muse muss her. Rettung naht in Form der jungen Viola de Lesseps, die das Theater liebt und sich, da es Frauen verboten ist, auf der Bühne zu stehen, unter dem Namen ,Thomas Kent' für die Hauptrolle in Shakespeares noch ungeschriebenen neuen Stück bewirbt. Wie in Was ibr wollt, für das Viola de Lesseps am Ende des Films Patin steht, ist die weibliche Hauptfigur also in vielen Szenen in Männerkleidern zu sehen.

Doch die Unterschiede zu Shakespeares Komödien sind auffällig. Denn im Gegensatz zu Was ibr wollt und Wie es euch gefällt, in denen die verkleidete Heldin von ihrem zukünftigen Liebhaber ja gerade nicht als Frau erkannt wird, und nur das Publikum um ihre wahre Identität weiß, ist Will im Film schnell eingeweiht, so dass die erotischen Spannungen zwischen den beiden von Anfang an in vorhersehbare, d.h. heterosexuelle Bahnen gelenkt werden. Der Spaß, den Shakespeares Stücke gerade aus den von den anderen Figuren falsch gedeuteten Signalen ziehen, fehlen in Shakespeare in Love fast völlig. Will verliebt sich nicht in Thomas, sondern in Viola, als er sie - auf der Suche nach Thomas Kent, dessen Gesicht er bis dahin noch gar nicht gesehen hat, - in ihren „Mädchenkleidern“, nämlich in einem festlichen Ballkleid sieht, ein Anblick, der in Was ibr wollt auf die Zeit nach der dramatischen Handlung verlegt wurde. Thomas Kent hat auch keine weiblichen Verehrerinnen, wobei sich hier allerdings auch kaum Gelegenheiten bieten, da Viola als Thomas ausschließlich in der Männerdomäne der Theaterwelt weilt.

Die Auflösung der Geschlechterambivalenz wird ebenfalls gänzlich anders vollzogen. Die Gegenüberstellung der Zwillinge in der letzten Szene von Was ibr wollt hat etwas Magisches und erinnert an mystische Darstellungen des alchemistischen Hermaphroditen. In Wie es euch gefällt bestimmt Rosalinde selbst, wann sie ihre Rolle als Ganymed aufgibt, und die Doppeldeutigkeiten, mit denen die Stücke gespielt haben, werden nie völlig aufgelöst. In Shakespeare in Love werden Männlein und Weiblein anhand ihres biologischen Geschlechts quasi naturbedingt ihren kulturell vorgesehenen Rollen zugeordnet. Viola verliert ihre Kurzhaarperücke und wird beim Liebesspiel mit Will ertappt - die nackte Wahrheit kommt ans Licht. Die ,natürlichen' Körper werden auf dem Höhepunkt des Films ihren ,authentischen' Rollen zugeführt: Viola spielt nicht mehr Romeo, die Rolle, die sie geprobt hat, sondern Julia; der boy actor wird durch seinen Stimmbruch endgültig zum Mann und damit als Julia unbrauchbar, und Will Shakespeare kann seine Liebe zu Viola in der Rolle des Romeo vor dem versammelten Publikum offenbaren - der Autor wird gleichgesetzt mit dem tragischen Helden. ${ }^{40}$

Eng mit diesem Handlungsverlauf verbunden ist die Privilegierung einer naturalistischen Schauspieltechnik durch den Film. Nachdem sie einer Aufführung von

40 Vgl. hierzu Traub und Iyengar. 
Zwei Herren aus Verona beigewohnt hat, beschwert sich Viola: „,Silvia' hat nicht viel Eindruck auf mich gemacht. Seine Finger waren rot vom Raufen und er sprach wie ein Schulbengel seine Lektion. Theaterliebe wird nie wie wahre Liebe, solange das Gesetz verlangt, dass unsere Heldinnen von schmalbrüstigen Knaben in Weiberkleidern gespielt werden müssen!“ Da die Filmzuschauer um den Auftritt von „Silvia' gebracht wurden, müssen sie Violas Kritik an dem anscheinend schlecht ausgebildeten boy actor zunächst einmal hinnehmen. Aber natürlich äußert Viola hier nicht nur Enttäuschung über eine miserable Einzelleistung. Es ist nicht einmal in erster Linie die Ungerechtigkeit, Frauen das Schauspielen zu verbieten, die hier angeklagt wird. Was Viola umtreibt, ist die nicht authentische Darstellung von Realität im elisabethanischen Theater, das „wahre Liebe“, was immer man sich darunter vorstellen mag, nicht ,natürlich`zu repräsentieren vermag. Damit vertritt sie selbstverständlich eine äußerst moderne Meinung, und die Zuschauer mögen in Viola eine Verbündete erkennen, eine Prophetin des ,method acting. ${ }^{6}{ }^{41} \mathrm{Im}$ Film kommt es schließlich zu einer Wette, die niemand anders als Königin Elisabeth selbst vorschlägt: „Gibt es ein Stück, das uns die wahrhafte Natur der Liebe zeigt?" Die Antwort heißt am Ende: Ja, aber nur wenn das Stück eine Tragödie von Shakespeare ist, und Männer und Frauen ihre authentischen, d.h. kulturell vorgeschriebenen Rollen spielen. Die Wette wird gewonnen, und die Kinozuschauer sind aufgefordert, diese Entwicklung als aufklärerischen Fortschritt zu lesen.

Natürlich ist der Film an vielen Stellen bewusst anachronistisch. Das macht seinen Charme aus und trägt viel zur Komik bei, wenn etwa die elisabethanische Theaterwelt durchgängig mit der amerikanischen Hollywood-Maschinerie verglichen wird. Dass die unterhaltsame und intelligente proto-feministische Liebesgeschichte, die Shakespeare in Love hauptsächlich erzählt, - Viola setzt sich durch und steht am Schluss als gefeierte Julia auf der Bühne -allerdings nur auf Kosten der komplexen und mehrdeutigen Gender-Spiele, wie wir sie in Shakespeares Komödien finden, funktioniert, ist auffällig. So mag zwar die Heldin in Männerkleidern rein äußerlich den cross-dressing comedies entsprungen sein (auch wenn man sich bemüht hat, die Schauspielerin Gwyneth Paltrow in einen möglichst unansehnlichen jungen Mann zu verwandeln), die Gemeinsamkeiten erübrigen sich damit jedoch weitgehend. Denn die Möglichkeit, mit der ,Doppelgeschlechtlichkeit ${ }^{6}$ seiner Heldin zu spielen, kostet der Film kaum aus. Und so mag man denn am Ende auch nicht so ganz glauben, dass diese Viola die Inspiration für $W$ as ibr wollt gewesen sein soll. Tatsächlich scheint Gautiers Roman aus dem frühen 19. Jahrhundert viel eher dem Zeitgeist der 1990er Jahre zu entsprechen.

41 Eine ähnlich teleologische Struktur haben auch die Filme Stage Beauty, in dem das Ende der boy actors am Beispiel des Schaupielers Edward Kynaston thematisiert wird, und The Libertine (basierend auf dem Theaterstück von Stephen Jeffreys), der u.a. von John Wilmots Faszination für die Schauspielerin Elizabeth Barry handelt, die durch ihre naturalistischen Darstellungen von Shakespeareheldinnen berühmt wurde. 
Gerade die Komödie ist jedoch traditionellerweise besonders dazu geeignet, innerhalb eines sicheren, da als künstlich und unrealistisch gekennzeichneten Rahmens kontroverse Themen zu behandeln oder sich auf Gedankenspiele einzulassen, die trotz aller Komik durchaus das Potential haben, an den Fundamenten festgefahrener hegemonialer Strukturen zu rütteln. Schließen möchte ich daher mit einem kurzen Abstecher zur wohl gelungensten Transvestiten-Komödie des 20. Jahrhunderts, Billy Wilders Some Like It Hot (Manche mögen's heiß) aus dem Jahre 1959. Der Film greift, wenn auch nicht mit explizitem Verweis auf Shakespeare, viele der Motive und Fragestellungen von Was ibr wollt und Wie es euch gefällt auf. Auch hier schlüpfen die Protagonisten - diesmal allerdings zwei Männer - aus Not in die Kleider und Rollen des anderen Geschlechts, um einer lebensbedrohlichen Situation zu entgehen. Auf der Flucht vor Gangstern tauchen die Musiker Joe und Jerry (gespielt von Tony Curtis und Jack Lemmon) in einer Damenkapelle unter. Den ersten Hinweis auf die Realitätsenthobenheit der nun folgenden Handlung erhalten die Kinozuschauer, als Jerry den von Joe vorgeschlagenen und sehr naheliegenden (Deck)namen Geraldine verwirft und sich stattdessen Daphne nennt - eine Referenz an die von Apoll begehrte Nymphe aus Ovids Metamorphosen, die sich, um dem verliebten Gott zu entkommen, in einen Lorbeerbaum verwandelt. Mit diesem Verweis auf die griechische Mythologie und ihre mannigfaltigen Transformationen wird dem Publikum signalisiert, dass mit der inszenierten Geschlechtsumwandlung der beiden Männer ein fantastisches Wunderland der unbegrenzten Möglichkeiten, ein Illyrien, betreten wird.

Als Josephine ist es Joe möglich, seiner Traumfrau Sugar Kane (Marilyn Monroe), ganz nahe zu sein, er kann sie aber in seiner Frauenrolle nicht offen umwerben. Joe befindet sich also in der gleichen Situation wie Viola, der Orsino zwar sein Herz ausschüttet, sie als Cesario aber zunächst anscheinend nicht als möglichen Liebespartner wahrnimmt. Joe versucht diesem Dilemma zu entkommen, indem er eine weitere männliche Rolle für sich kreiert, den bebrillten Jungmillionär Junior. Der leidenschaftlichste Kuss des Films findet jedoch zwischen Sugar und Josephine statt, und Sugar gibt am Ende ihre Liebe zu ,Junior ${ }^{`}$ auf, um sie Joe (oder auch Josephine) zu schenken.

Für Jerry ist es zunächst schwierig, seinen Part überzeugend zu spielen, und er muss sich mit Hilfe von Autosuggestion auf Kurs halten („Ich bin ein Mädchen, ich bin ein Mädchen"). Doch schon bald geht er so sehr in seiner neuen Identität auf - von Rolle mag man schon gar nicht mehr sprechen -, dass er die Avancen des lüsternen Millionärs Osgood tatsächlich erwidert und seinem Freund Joe erfreut von seinen Hochzeitsplänen berichtet. Joes Einwände werden mit bestechender Logik einer nach dem anderen für null und nichtig erklärt: ,Jerry, du kannst doch Osgood nicht heiraten!“ - „Meinst du, er ist zu alt für mich?“ Dass Joe eigentlich nur darauf hinauswill, dass das Geschlecht (allein schon aus gesetzlichen Gründen) ein Hindernisgrund sein könnte, darauf scheint Daphne gar nicht gekommen zu sein. Auch auf Joes verzweifeltes: „Du bist ein Mann! Und warum 
sollte ein Mann einen Mann heiraten?“ antwortet Daphne nur: „Aus Sicherheitsgründen!“ Ähnlich wie Viola, die von „Ich bin nicht, was ich spiele“ (1.5.176) ${ }^{42}$ schließlich bei „Ich bin nicht, was ich bin“ (3.1.139)43 ankommt und kurz vor einer Identitätskrise steht, hat Jerry sich in seiner neuen Rolle so eingenistet, dass er anscheinend gar nicht mehr zurückkann oder -will: „Ich bin ein Mann, ich bin ein Mann, ich wollte ich wäre tot. Ich bin ein Mann.“

Der Film endet mit einer Parallel-Szene. Diesmal ist es Daphne, die ihrem ,Verlobten'klarzumachen versucht, warum sie nicht heiraten können. Und wieder werden alle Einwände für trivial erklärt und einfach beiseite geschoben. Osgood und wenn wir ehrlich sind, auch wir Zuschauer - wollen die unterhaltsame, extravagante Kunstfigur Daphne nicht gegen den viel langweiligeren Jerry eintauschen. Genau wie Ganymed oder Cesario ist Jerrys transvestiertes Ich nicht einfach nur ein Männerkörper im Damenkostüm, sondern eine ganz neue Figur mit einer eigenen Persönlichkeit, die als Resultat der Kostümierung entstanden ist. ${ }^{44}$ Dass sogar Daphnes ultimatives Geständnis „Ich bin ein Mann!“, begleitet vom AbreiBen der Perücke und vom Wechsel der Stimmlage, nur mit einem dahingeworfenen „Na und? Niemand ist vollkommen!“ („Well, nobody’s perfect!“) gewürdigt wird, lässt für einen Moment, abgesichert von der Künstlichkeit des Genres und der Absurdität der Situation, die Idee aufscheinen, dass biologisches Geschlecht für das Begehren irrelevant ist, wenn die performance der (Gender-)Rolle stimmt. Um Simone de Beauvoir gegen den Strich zu lesen: Jerry wurde zwar nicht als Frau geboren, aber Daphne wurde eine.

42 „I am not that I play.“ (1.5.185)

43 „I am not what I am.“ (3.1.143)

44 Zu Manche mögen's heiß vgl. Garber 7 und Bell-Metereau 54-65. 


\section{Verwendete Literatur}

\section{Primärliteratur}

Gautier, Théophile. Mademoiselle de Maupin. 1835. Übers. (Hans Henning Baron Voigt) Alastair. München: Knaur, 1987.

Shakespeare, William. Antonius und Kleopatra: zweisprachige Ausgabe. Neu übers. und mit Anm. versehen von Frank Günther. Mit einem Essay und Literaturhinweisen von Bernhard Klein. Cadolzburg: Ars Vivendi, 2000.

Shakespeare, William. Antony and Cleopatra. The Arden Shakespeare. Hg. John Wilders. London: Routledge, 1995.

Shakespeare, William. As You Like It. The Arden Shakespeare. Hg. Juliet Dusinberre. London: Thomson, 2006.

Shakespeare, William. The Sonnets - Die Sonette: Englisch und in ausgewählten deutschen Versübersetzungen. Hg. Raimund Borgmeier. Stuttgart: Reclam, 1974.

Shakespeare, William. Twelfth Night. The Arden Shakespeare. Hg. J. M. Lothian und T. W. Craik. London: Thomson, 2005.

Shakespeare, William. Was ibr wollt: zweisprachige Ausgabe. Neu übers. und mit Anm. versehen von Frank Günther. Mit einem Essay und Literaturhinweisen von Christa Jansohn. Cadolzburg: Ars Vivendi, 2001.

Shakespeare, William. Wie es euch gefällt: zweisprachige Ausgabe. Neu übers. und mit Anm. versehen von Frank Günther. Mit einem Essay und Literaturhinweisen von Andreas Mahler. Cadolzburg: Ars Vivendi, 2002.

\section{Sekundärliteratur}

Barbin, Herculine und Michel Foucault. Über Hermaphrodismus: der Fall Barbin. Frankfurt a. M.: Suhrkamp, 1998.

Beauvoir, Simone de. Das andere Geschlecht: Sitte und Sexus der Frau. 9. Aufl. Übers. Uli Aumüller und Grete Osterwald. Reinbek bei Hamburg: Rowohlt, 2008.

Bell-Metereau, Rebecca. Hollywood Androgyny. New York: Columbia Univ. Pr., 1985.

Bulman, James C. „Queering the Audience: All-Male Casts in Recent Productions of Shakespeare." A Companion to Shakespeare and Performance. Hg. Barbara Hodgdon und W. B. Worthen. Malden, Mass.: Blackwell, 2005, 564-587.

Butler, Judith. Gender Trouble: Feminism and the Subversion of Identity. 2. Aufl. New York: Routledge, 1999.

Duncker, Patricia. „Introduction.“ Théophile Gautier. Mademmoiselle de Maupin. Hg. und Übers. Helen Constantine. London: Penguin, 2005, xi-xxviii. 
Franceschina, John. Homosexualites in the English Theatre: From Lyly to Wilde. Westport, Conn.: Greenwood Pr., 1997.

Freud, Sigmund. „Der Untergang des Ödipuskomplexes.“ Gesammelte Werke. 9. Aufl. Hg. Anna Freud u.a. Bd. 13. Frankfurt a. M.: Fischer, 1987, 395-402.

Freud, Sigmund. „Die Weiblichkeit.“ Gesammelte Werke. 3. Aufl. Hg. Anna Freud u.a. Bd. 15. Frankfurt a. M: Fischer, 1961, 119-45.

Garber, Marjorie. Vested Interests: Cross-Dressing and Cultural Anxiety. New York: Routledge, 1992.

Greenblatt, Stephen. „About that Romantic Sonnet.“ New York Times, 6. Febr. 1999.

Greenblatt, Stephen. „Fiction and Friction.“ Shakespearean Negotiations: The Circulation of Social Energy in Renaissance England. Oxford: Clarendon Pr., 1997, 66-93.

Howard, Jean. The Stage and Social Struggle in Early Modern England. London: Routledge, 1994.

Howards, Tony. Women as Hamlet. Performance and Interpretation in Theatre, Film and Fiction. Cambridge: Cambridge Univ. Pr., 2007.

Iyengar, Sujata. „Shakespeare in Heterolove.“ Literature/Film Quarterly 29, 2 (2001), 122-7.

Jardine, Lisa. „,Make thy doublet of changeable taffeta': Dress Codes, Sumptuary Law and ,Natural" Order." Still Harping on Daughters: Women and Drama in the Age of Shakespeare. Sussex: Harvester Pr., 1983, 141-168.

Masten, Jeffrey. „Textual Deviance: Ganymede’s Hand in As You Like It.“ Field Work: Sites in Literary and Cultural Studies. Hg. Marjorie Garber u.a. New York: Routledge, 1996, 153-63.

Orgel, Stephen. Impersonations: The Performance of Gender in Shakespeare's England. Cambridge: Cambridge Univ. Pr., 1996.

Sedinger, Tracey. „If sight and shape be true': The Epistemology of Crossdressing on the London Stage." Shakespeare Quarterly 48, 1 (1997), 63-79.

Smith, Bruce R. Homosexual Desire in Shakespeare's England: A Cultural Poetics. Chicago: The Univ. of Chicago Pr., 1991.

Stallybrass, Peter. „Transvestism and the ,Body Beneath': Speculating on the Boy Actor." Erotic Politics: Desire and the Renaissance Stage. Hg. Susan Zimmerman. New York: Routledge, 1992, 64-83.

Traub, Valerie. „The Sonnets: Sequence, Sexuality, and Shakespeare's Two Loves." A Companion to Shakespeare's Works. Hg. Richard Dutton und Jean E. Howard. Bd. 4: The Poems, Problem Comedies, Late Plays. Malden, Mass.: Blackwell, 2003, 275-301. 
Filme

I'm Not There. Regie Todd Haynes. 2007. DVD. Ufa, 2008.

The Libertine. Regie Laurence Dunmore. 2005. DVD. Entertainment in Video, 2006.

Manche mögen's heiss. Regie Billy Wilder. 1959. DVD. Focus Edition, 2006.

Shakespeare in Love. Regie John Madden. 1998. DVD. Universal Pictures, 2006.

Stage Beanty. Regie Richard Eyre. 2004. DVD. Universum Film, 2007.

Twelfth Night. Regie Trevor Nunn. 1996. DVD. Entertainment in Video, 2001. 